\title{
DIFERENÇAS SOCIOECONÔMICAS REGIONAIS NOS MUNICÍPIOS DE MATO GROSSO DO SUL
}

Paulo Henrique Hoeckel ${ }^{1}$ Maria Cristina Vezzu Costa ${ }^{2}$

Resumo: Este artigo teve como objetivo analisar a possível existência de diferenças regionais quanto ao desenvolvimento socioeconômico nos municípios de Mato Grosso do Sul. Para tanto, utilizou-se o ferramental da Análise Exploratória de Dados Espaciais e as variáveis do Índice de Desenvolvimento Humano Municipal para os anos de 1991, 2000 e 2010 e o Índice Firjan de Desenvolvimento Municipal para o ano de 2016, buscando verificar a existência de clusters espaciais. Os principais resultados mostram que os municípios sofrem influência dos municípios vizinhos, apresentando disparidades regionais em relação a distribuição espacial do desenvolvimento socioeconômico, o que mostra a necessidade de políticas públicas específicas para melhorar esse desequilíbrio regional.

Palavras-chave: Desenvolvimento socioeconômico. Cluster espacial. Análise Exploratória de Dados Espaciais. Municípios. Mato Grosso do Sul.

\section{REGIONAL SOCIOECONOMIC DIFFERENCES IN THE MUNICIPALITIES OF MATO GROSSO DO SUL, BRAZIL}

Abstract: This study aimed to analyze the possible existence of regional differences in socioeconomic development in the municipalities of Mato Grosso do Sul. For this, we used the tool of Exploratory Spatial Data Analysis and the Municipal Human Development Index variables for the years 1991, 2000, and 2010 and the Municipal Firjan Development Index for 2016, seeking to verify the existence of spatial clusters. The main results show that municipalities are influenced by neighboring municipalities, presenting regional disparities in relation to the spatial distribution of socioeconomic development, which shows the need for specific public policies to improve this regional imbalance.

Keywords: Socioeconomic development. Spatial cluster. Exploratory Spatial Data Analysis. Municipalities. Mato Grosso do Sul.

\section{DIFERENCIAS SOCIOECONÓMICAS REGIONALES EN LOS MUNICIPIOS DE MATO GROSSO DO SUL}

Resumen: Este artículo tuvo como objetivo analizar la posible existencia de diferencias regionales en términos de desarrollo socioeconómico en los municipios de Mato Grosso do Sul. Para ello, se utilizaron la herramienta de Análisis de Datos Espaciales Exploratorios y las variables Índice de Desarrollo Humano Municipal para los años 1991, 2000 y 2010, y el Índice de Desarrollo Municipal Firjan para el año 2016, buscando verificar la existencia de agrupaciones espaciales. Los principales resultados muestran que los municipios están influenciados por los municipios vecinos, presentando disparidades regionales en relación con la distribución espacial

\footnotetext{
1 Universidade Federal da Grande Dourados, Departamento de Economia, Dourados, Brasil, ph.hoeckel@gmail.com, https://orcid.org/0000-0002-1017-8975

2 Universidade Federal da Grande Dourados, Departamento de Economia, Dourados, Brasil, racco.dourados@hotmail.com,https://orcid.org/0000-0001-5363-8479
} 
del desarrollo socioeconómico, lo que demuestra la necesidad de políticas públicas específicas para mejorar este desequilibrio regional.

Palabras clave: Desarrollo Socioeconómico. Clúster espacial. Análisis exploratorio de datos espaciales. Condados. Mato Grosso do Sul.

\section{Introdução}

Quando se analisa o quão rica é uma nação, estado ou município, se está preocupado com o nível de renda e se o mesmo está tendo crescimento. Porém, para saber mais informações sobre variáveis qualitativas a respeito de qual é a condição de vida da população residente, precisa-se analisar mais detalhadamente esse nível de renda, de modo, a saber, de que forma o mesmo é distribuído e como ele interfere nas condições de saúde e educação da população (MOLDAU, 1998).

Assim, quando o interesse está voltado para questões sociais é necessário avaliar o desenvolvimento econômico de uma região e não só o seu crescimento. Nesse sentido, de acordo com Vidigal, Amaral e Silveira (2012), o desenvolvimento socioeconômico pode ser analisado através de alguns índices, sendo o mais importante deles o Índice de Desenvolvimento Humano (IDH). Composto por três indicadores (saúde, educação e renda), que representam a oportunidade de uma sociedade de ter vida longa e saudável, de ter acesso ao conhecimento e de ter comando sobre os recursos de forma a garantir um padrão de vida digno. O Brasil foi um dos países pioneiros ao adaptar e calcular um IDH subnacional para todos os municípios brasileiros, com dados do Censo Demográfico, criando o Índice de Desenvolvimento Humano Municipal (IDHM).

O desenvolvimento socioeconômico está atrelado às mudanças qualitativas nas condições de vida da sociedade, na qualidade e eficiência das instituições e também das estruturas produtivas do país. Portanto, tem como elemento principal, a melhoria no nível de bem-estar. Pensar em desenvolvimento econômico é pensar em modernidade, em mão de obra qualificada e valorizada, em inovações tecnológicas produzidas por empresas domésticas, em eficiência da estrutura produtiva e também governamental (SIEDENBERG, 2003).

Conforme Rocha (1998), o Brasil, seguindo esse contexto, é marcado pelas intensas desigualdades sociais e regionais caracterizadas pela miséria, a ignorância, a enfermidade, dentre outros. A sociedade é vítima de várias formas de privação de liberdade básica para sobreviver que lhes impede do processo de desenvolvimento. 
O desenvolvimento socioeconômico implica, deste modo, em mudanças no quadro político, institucional, econômico e social.

Como resultado desse processo, segundo Sicsú e Castelar (2009), os municípios têm demonstrado desafios para serem enfrentados, relacionados à questão socioeconômica, às discrepâncias em relação à renda do trabalhador assalariado por região do país, bem como no sentido de buscar alternativas concretas para melhoria da qualidade de vida dos grandes centros urbanos que concentram elevado percentual da população brasileira. Esses desafios refletem a possibilidade de existirem diferenças regionais no tocante ao desenvolvimento socioeconômico.

Logo, é necessário avaliar de modo mais desagregado possível o desenvolvimento socioeconômico regional, ou seja, a nível municipal, para evidenciar possíveis desequilíbrios regionais, podendo assim contribuir para políticas públicas voltadas especificamente na tentativa de saná-los. Dentro dessa perspectiva, procura-se analisar o desenvolvimento socioeconômico no estado de Mato Grosso do Sul (MS), que conta com 79 municípios, tendo apresentado um Produto Interno Bruto (PIB) de R \$ 91,87 bilhões em 2016 e um PIB per capita de $\mathrm{R} \$$ 34.247,79. O IDH de MS é de 0,729 (considerado alto), se comparado com outros estados do país, ele ocupa a décima posição (IBGE, 2010).

Os três municípios de MS com melhor IDHM são, respectivamente, Campo Grande (0,784), Chapadão do Sul $(0,754)$ e Dourados $(0,747)$. Enquanto entre os três piores colocados estão Japorã $(0,526)$, Paranhos $(0,588)$ e Coronel Sapucaia $(0,589)$, conforme dados do Instituto de Pesquisa Econômica Aplicada (IPEA, 2018). A diferença nos indicadores, certo modo, evidência a possibilidade de diferenças regionais quanto ao desenvolvimento socioeconômico, dessa forma é importante analisar a existência desse fenômeno, de modo mais minucioso, para que, caso ele se verifique, políticas econômicas possam ser propostas visando sanar esse problema.

Tais fatores justificam a importância desta pesquisa, além de verificado que nacionalmente existem um número considerável de estudos relacionados a municípios e microrregiões, a nível federal ou para outros estados (PAES DE BARROS; MENDONÇA, 1997; MOLDAU, 1998; ROCHA, 1998; MORAES, 2000; SIEDENBERG, 2003; RODRIGUES; SIMÕES, 2004; REZENDE; SLOMSKI; CORRAR, 2005; SHIKIDA, 2008; PERES et al., 2008; VIDIGAL; AMARAL; SILVEIRA, 2012; REIS; SILVEIRA; BRAGA, 2013), mas pouquíssimos trabalhos 
(CONSTANTINO; PEGORARE; DA COSTA, 2016; LIMA; PIFFER; OSTAPECHEN, 2016) que abordem, de alguma forma, a questão do desenvolvimento socioeconômico para os municípios sul mato-grossenses e quais os impactos que ocorreram ao longo do período analisado.

Portanto, o objetivo do presente estudo é analisar a possível existência de diferenças regionais quanto ao desenvolvimento socioeconômico nos municípios de Mato Grosso do Sul. Mais especificamente buscou-se analisar os dados estatísticos obtidos para maior compreensão de indicadores ligados à educação, saúde e renda, para que seja possível estabelecer um perfil regional, com as características estruturais dos municípios e suas principais demandas.

Para tanto, o presente estudo utiliza o IDHM dos municípios de MS, considerando os Censos Demográficos de 1991, 2000, 2010 e para o ano de 2016 é utilizado o Índice Firjan de Desenvolvimento Municipal (IFDM), para analisar o desenvolvimento socioeconômico nesses municípios e, também, com o intuito de avaliar a existência de possíveis diferenças regionais.

O nível de desenvolvimento socioeconômico de um município, região e estado, demonstra a capacidade que os mesmos possuem de distribuir o crescimento da renda de forma a melhorar a qualidade de vida da sua população. $A$ existência de diferenças regionais pode impactar em gerar regiões mais desenvolvidas, com municípios mais ricos, enquanto municípios mais pobres podem padecer por mais tempo (desenvolvimento mais demorado), assim a localização de um município pode ser muito importante dada a possibilidade de acesso a saúde, empregos com melhor remuneração e educação de seus municípios vizinhos (transbordamentos) ${ }^{3}$.

\section{Fundamentos conceituais: o IDHM e o IFDM}

Conforme Lima, Piffer e Ostapechen (2016), o desenvolvimento socioeconômico se refere a qualquer prática que relaciona um aspecto que afeta tanto a ordem econômica como social, representa a aplicação das riquezas de modo que melhore a qualidade de vida das pessoas no âmbito da saúde, educação, alimentação, renda e outros indicadores de bem-estar social. Nesse sentido, o desenvolvimento econômico é definido pela existência de crescimento econômico contínuo, em ritmo superior ao crescimento demográfico, envolvendo mudanças de

\footnotetext{
${ }^{3} \mathrm{O}$ fato de uma região se beneficiar por estar próxima, ser vizinha, de regiões desenvolvidas é conhecida como spillovers, traduzida como transbordamentos.
} 
estruturas e melhoria de indicadores econômicos e sociais. Para que haja desenvolvimento econômico é preciso que tenha crescimento, que é calculado através de indicadores referentes a quantidades, medido pela variação do PIB.

Portanto, o desenvolvimento econômico compreende um fenômeno de longo prazo, implicando no fortalecimento da economia nacional via acumulação de capital e incorporação de conhecimento ou progresso tecnológico à produção, na ampliação da economia de mercado e na elevação geral da produtividade (SICSÚ; CASTELAR, 2009). Dessa forma, um país, estado ou município é considerado desenvolvido quando possui bons indicadores de desenvolvimento socioeconômico, sendo o IDH um dos principais indicadores utilizados para refletir a qualidade de vida da população residente.

O IDH, é uma medida composta de indicadores de saúde, educação e renda, foi criado em 1990, para o relatório de Desenvolvimento Humano do Programa das Nações Unidas para o Desenvolvimento. Com objetivo de servir como uma referência para o nível de desenvolvimento humano de uma determinada localidade. O índice varia de 0 (valor mínimo) a 1(valor máximo), sendo que a composição do IDH assume que para viver a vida que desejam, as pessoas precisam pelo menos ter a possibilidade de levar uma vida longa e saudável, acesso a conhecimento e a oportunidade de desfrutar de um padrão de vida digno (SEN, 2000).

Um bom indicador para avaliar a questão socioeconômica a nível municipal é o IDHM criado em 1998, o índice ajusta o IDH para realidade dos municípios e regiões metropolitanas e reflete as especificidades e desafios regionais no alcance do desenvolvimento humano da população residente, preocupado com as dimensões educação, saúde (longevidade) e renda (PNUD, 2013). Com base na importância desse indicador para se avaliar o desenvolvimento, sendo utilizado e reconhecido mundialmente, o presente estudo utiliza o IDHM, de modo que este adequa a metodologia global ao contexto brasileiro e à disponibilidade de indicadores nacionais, especificamente no presente estudo, os municípios de MS.

O IDHM é um índice composto que agrega três das mais importantes dimensões do desenvolvimento humano: a oportunidade de viver uma vida longa e saudável, de ter acesso ao conhecimento e ter um padrão de vida que garanta as necessidades básicas, representadas pela saúde, educação e renda. A primeira dimensão, relacionado a vida longa e saudável (longevidade) é medida pela expectativa de vida ao nascer, calculada por método indireto a partir dos dados dos Censos Demográficos do IBGE. Esse indicador mostra o número médio de anos que 
as pessoas viveriam a partir do nascimento, mantidos os mesmos padrões de mortalidade observados no ano de referência (PNUD, 2013).

Assim como no IDH global, o IDHM Educação é uma composição de dois indicadores: um indicador fornece informação sobre a situação educacional da população adulta e o outro referente à população em idade escolar (jovens). Entretanto, as variáveis são outras. No caso da população adulta, a média de anos de estudo de pessoas de 25 anos ou mais, tal como é medido no IDH Global, não pode ser obtida das informações do Censo 2010 e foi substituída pela proporção da população adulta de 18 anos ou mais que concluiu o ensino fundamental. Este indicador permite uma boa avaliação do nível de carência da população adulta em relação à escolaridade considerada básica (nível fundamental). No caso da população jovem, a metodologia aplicada pelo IDH Global a partir de 2010 - a expectativa de vida escolar - é uma medida de retenção das pessoas na escola, independentemente da repetência, e inclui o ensino superior (PNUD, 2013).

A adaptação do IDHM para os contextos nacional e municipal foi feita com uma combinação de quatro indicadores que permitem verificar até que ponto as crianças e os jovens estão frequentando e completando determinados ciclos da escola. O sub índice resultante, o fluxo escolar da população jovem, é a média aritmética do percentual de crianças de 5 a 6 anos frequentando a escola, do percentual de jovens de 11 a 13 anos frequentando os anos finais do ensino fundamental (6ำ ao $9^{\circ}$ ano), do percentual de jovens de 15 a 17 anos com ensino fundamental completo e do percentual de jovens de 18 a 20 anos com ensino médio completo (PNUD, 2013).

Por fim, o padrão de vida é medido pela renda municipal per capita (em $R \$$ ), ou seja, a renda média de cada residente de determinado município. Portanto, é a soma da renda de todos os residentes, dividida pelo número de pessoas que moram no município, inclusive crianças e pessoas sem registro de renda. Os dados são do Censo Demográfico do IBGE. Os três componentes acima são agrupados por meio da média geométrica, resultando no IDHM (PAES DE BARROS; MENDONÇA, 1997).

O IFDM, criado em 2008 pela Federação das Indústrias do Estado do Rio de Janeiro, abrange três dimensões do desenvolvimento em sua composição: Educação, Saúde, Emprego e Renda. É calculado anualmente, desde sua criação, para todos os municípios brasileiros. Cada uma de suas três dimensões é 
sintetizada em subíndices (IFDM Educação, IFDM Saúde e IFDM Emprego e Renda) cujos valores também variam de 0 a 1.

Desde 2014, a metodologia do IFDM foi aprimorada para captar os novos desafios do desenvolvimento brasileiro para esta nova década. O principal incremento foi situar o Brasil no mundo. A nova metodologia buscou padrões de desenvolvimento encontrados em países mais avançados, utilizando-os como referência para os indicadores municipais. Outro ponto importante foi a atualização de metas e parâmetros nacionais. Neste caso, o ano de referência deixou de ser 2000 e passou a ser 2010 (FIRJAN, 2018).

De leitura simples, o índice varia de 0 (mínimo) a 1 ponto (máximo) para classificar o nível de cada localidade em quatro categorias: baixo (de 0 a 0,4 ), regular $(0,4$ a 0,6$)$, moderado (de 0,6 a 0,8$)$ e alto $(0,8$ a 1$)$ desenvolvimento. Ou seja, quanto mais próximo de 1, maior o desenvolvimento da localidade. Dessa forma, é possível determinar com precisão se a melhora ocorrida em determinado município decorre da adoção de políticas específicas ou se o resultado obtido é apenas reflexo da queda dos demais municípios. Por conseguinte, a ênfase da leitura não deve apenas se restringir a uma questão de posição no ranking, mas sim de se verificar se, de fato, houve progresso de um determinado município ou região, em dado período de tempo (FIRJAN, 2018).

\section{Metodologia}

Análise Exploratória de Dados Espaciais (AEDE)

Este trabalho utiliza como ferramental metodológico a análise espacial. A visualização gráfica é a etapa inicial para descrever a distribuição espacial dos dados. Posteriormente, se torna necessário verificar a aleatoriedade dos dados espaciais, de modo que, se os valores do atributo em uma região não dependem desse atributo nas regiões vizinhas, a autocorrelação espacial não é verificada. Conforme Hoeckel, Casagrande e Santos (2019), a existência de autocorrelação espacial configura a dependência espacial, a qual retrata uma situação em que os valores observados em uma região dependem dos valores observados nas regiões vizinhas (gerando formação de clusters espaciais). Para implementar a AEDE é preciso, ponto de partida, definir uma matriz de pesos espaciais. 


\section{Matriz de Pesos Espaciais}

A matriz de pesos espaciais $W^{4}$ é uma matriz não-negativa de constantes conhecidas, usadas para realizar ponderação da influência que as regiões exercem entre si, sendo baseada na associação, que pode ser definida de acordo com a vizinhança, a distância tanto geográfica quanto socioeconômica, bem como a combinação disso (ALMEIDA, 2004).

Almeida (2004) considera que a forma mais simples para definir uma matriz de pesos espaciais é utilizar uma matriz binária de vizinhança. Assim tem-se que: se duas regiões são vizinhas, ou seja, partilham de uma fronteira, atribui-se o valor unitário, caso contrário o valor nulo, formalmente tem-se:

$$
\begin{aligned}
& w_{i j}=1, \text { se } i \text { e } j \text { são contíguos (vizinhos) } \\
& w_{i j}=0, \text { se } i \text { e } j \text { não são contíguos (vizinhos) }
\end{aligned}
$$

Por convenção, $w_{i i}=0$, ou seja, nenhuma região $i$ pode ser vizinha dela mesma.

Para definir vizinhança ou contiguidade, conforme Almeida (2004), é preciso adotar um conceito de fronteira geográfica, que pode ocasionar em erro de medidas por ser realizado mediante observação de um mapa (representação abstrata da real configuração geográfica). Levando em conta esses erros de medidas, e em alusão ao movimento de peças de um tabuleiro de xadrez, a convenção de contiguidade é dita ser rainha, caso, além das fronteiras com extensão diferente de zero, puderem ser considerados os vértices (nós), na visualização de um mapa, como contíguos. Caso apenas fronteiras físicas com extensão diferente de zero entre as regiões sejam levadas em conta, a convenção de contiguidade é considerada como torre. Essas convenções podem ser visualizadas na Figura 1, apresentada abaixo.

Figura 1. Matrizes de contiguidade Torre e Rainha

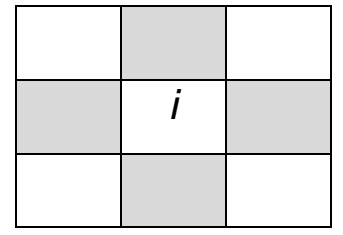

Torre

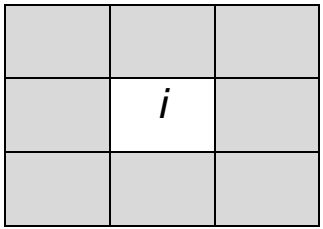

Rainha

Fonte: Adaptado de Almeida (2004).

\footnotetext{
${ }^{4}$ A matriz de pesos espaciais é uma matriz quadrada $(n \times n)$ que contém os pesos espaciais de cada unidade sobre outra. Assim, o elemento wij indica o peso espacial que a unidade $j$ exerce sobre a unidade $i$. Quando a matriz $W$ é construída, independente do critério utilizado, ela é tratada como um fator exógeno (HOECKEL; CASAGRANDE; SANTOS, 2019).
} 
Apesar das duas convenções, torre e rainha, serem as mais utilizadas na literatura, Almeida (2004) destaca que elas apresentam uma desvantagem, dado que não é garantida uma conectividade balanceada, uma vez que haja regiões com grande área com muitos vizinhos, ao passo que existirão regiões com pouca área e com poucos vizinhos.

Para superar esse problema, adota-se a matriz $k$ vizinhos mais próximos. Trata-se de uma matriz binária de contiguidade cuja convenção de vizinhança é baseada na distância geográfica (ALMEIDA, 2004). Pode-se formalizar a matriz $k$ da seguinte maneira:

$$
\begin{aligned}
& w_{i j}=1, \text { se } d_{i j} \leq \bar{d} \\
& w_{i j}=0, \text { se } d_{i j} \geq \bar{d}
\end{aligned}
$$

em que $\bar{d}$ é um valor de distância crítico.

A vantagem dessa convenção, conforme Almeida (2004), é combater o desbalanceamento da conectividade de uma matriz, pois todas as unidades espaciais terão o mesmo número de vizinhos cada uma. Uma vantagem comum a todas as matrizes de pesos espaciais do tipo binário é a possibilidade de definir vizinhos de ordens superiores.

A escolha da matriz de pesos espaciais é muito importante em uma AEDE, uma vez que os resultados da análise são sensíveis a tal seleção ${ }^{5}$.

\section{Índice de Moran Global}

O índice utilizado para verificar a autocorrelação espacial é o Índice de Moran, ele utiliza um único valor como medida de associação espacial para um conjunto de dados que é útil na caracterização da região de estudo, e através das análises locais busca-se testar a presença de diferenças espaciais existentes (ARAUJO; URIBE; JOHANN, 2014).

O Índice de Moran Local foi proposto por Anselin (1999) como uma ferramenta estatística para testar a autocorrelação local e para detectar objetos espaciais com influência no indicador Moran Global.

Ao calcular a autocorrelação espacial usando a estatística I de Moran obtémse a indicação formal do grau de associação linear entre os vetores de valores observados no tempo $t\left(z_{t}\right)$ e a média ponderada dos valores da vizinhança, ou dos

\footnotetext{
${ }^{5}$ Para mais informações sobre quais as melhores formas de escolha da matriz de pesos espaciais e demais formalizações das mesmas ver Anselin (1999).
} 
lags espaciais $\left(W z_{t}\right)$. Os valores de I maiores (ou menores) do que o valor esperado $E(I)=-1 /(n-1)$ demonstram a existência de autocorrelação positiva (ou negativa). Assim, o índice de Moran presta-se a um teste cuja hipótese nula é de independência espacial e neste caso o valor seria zero (ALMEIDA, 2004).

Conforme Almeida (2004), a autocorrelação espacial positiva revela a existência de uma similaridade entre os valores do atributo estudado e a localização espacial. A autocorrelação espacial negativa revela, por sua vez, que existe uma dissimilaridade entre os valores do atributo considerado a sua localização espacial.

A estatística I de Moran algebricamente é dada por:

$$
I_{i}=\left(\frac{n}{s_{0}}\right)\left(\frac{Z_{t}^{\prime} W Z_{t}}{Z_{\prime}{ }^{\prime} Z_{t}}\right) \quad t=1,2, \ldots, n
$$

onde $Z_{t}$ é o vetor de $n$ observações para o ano $t$ na forma de desvio em relação à média, $W$ é a matriz de pesos espaciais, sendo que os elementos $w_{i i}$ na diagonal são iguais a zero, enquanto que os elementos $w_{i j}$ indicam a forma como a região $i$ está espacialmente conectada com a região $j$. O termo $S_{0}$ é um escalar igual à soma de todos os elementos de $W$. A normalidade das linhas da matriz de pesos espaciais ${ }^{6}$, ou seja, quando os elementos de cada linha somam 1, altera a expressão (1) para a seguinte forma:

$$
I_{i}=\frac{Z^{\prime} W Z}{Z^{\prime} Z}
$$

Os indicadores globais de autocorrelação espacial, como o índice de Moran, fornecem um único valor como medida da associação espacial para todo o conjunto de dados, o que é útil na caracterização da região de estudo como um todo (ARAUJO; URIBE; JOHANN, 2014).

\section{Índice Local de Associação Espacial (LISA)}

De acordo com Almeida (2004), a estatística espacial local foi desenvolvida com o objetivo de qualificar o grau de associação espacial que cada localização do conjunto amostral está submetida em função de um modelo de vizinhança preestabelecida, denominada de Indicadores Locais de Associação Espacial (LISA).

Enquanto os indicadores globais, como o índice de Moran, fornecem um único valor como medida da associação espacial para todo o conjunto de dados, os indicadores locais produzem um valor específico para cada objeto, permitindo assim, a identificação de agrupamentos de objetos com valores de atributos semelhantes

\footnotetext{
${ }^{6}$ Para mais detalhes acerca da composição metodológica da matriz de pesos espaciais ver Almeida (2012) e Hoeckel, Casagrande e Santos (2019).
} 
(clusters), objetos anômalos (outliers) e de mais de um regime espacial (ANSELIN, 1999).

A estatística I de Moran local (LISA) pode ser obtida, conforme Anselin (1999), pela seguinte equação:

$$
I_{i}=\frac{\left(y_{i}-\bar{y}\right) \sum_{j} w_{i j}\left(y_{j}-\bar{y}\right)}{\sum_{i} w_{i j}\left(y_{i}-\bar{y}\right)^{2} / n}
$$

em que $z_{i}$ e $z_{j}$ são variáveis padronizadas e a somatória entre $j$ é tal que somente os valores dos vizinhos $j$ pertencente a $J_{i}$ são incluídos. O conjunto $J_{i}$ abrange os vizinhos da observação $i$.

O indicador LISA provê uma indicação do grau de agrupamento dos valores similares em torno de uma observação, identificando clusters espaciais, estatisticamente significantes. Tais clusters são divididos em quatro tipos de associação espacial, sendo: Alto-Alto (AA), Baixo-Baixo (BB), Alto-Baixo (AB) e Baixo-Alto (BA). Os agrupamentos AA e BB indicam a ocorrência de autocorrelação espacial positiva, enquanto que os agrupamentos $A B$ e BA denotam a presença de autocorrelação espacial negativa (ALMEIDA, 2012).

\section{Dados e área de estudo}

A base de dados é constituída pelo IDHM dos 79 municípios do estado de MS, oriundos do Censo Demográfico do IBGE, para os anos de 1991, 2000 e 2010. Com a finalidade de analisar o padrão mais recente do desenvolvimento humano, utilizou-se o IFDM para o ano de $2016^{7}$, obtido junto a Firjan, com as dimensões emprego e renda, saúde e educação. O Shapefile utilizado, que é um formato de arquivo contendo os dados geoespaciais em forma de vetor usado por Sistemas de Informações Geográficas (SIG), com os dados georeferenciados dos municípios é oriundo do IPEA. Para realizar as estimações e construções de mapas (formação de clusters $^{8}$ ) foram utilizados os softwares estatísticos IpeaGeo ${ }^{9}$ e Geoda ${ }^{10}$.

O estado de MS fica localizado no sul da Região Centro-Oeste do Brasil, o mesmo limita-se com cinco estados brasileiros (Mato Grosso, Goiás, Minas Gerais,

\footnotetext{
7 Para o município de Ribas do Rio Pardo foi utilizado a informação do IFDM de 2015, como proxy para o ano de 2016, pois a informação para 2016 não estava disponível para o mesmo (Emprego e Renda de 2015 também).

${ }^{8}$ Do inglês cluster significa grupo ou aglomerado, que consiste em algo fracamente ou fortemente ligados que atuam em proximidade. No caso do presente estudo, refere-se a grupo de municípios.

${ }^{9}$ Criado pela Assessoria de Métodos Quantitativos do Ipea - ASMEQ, o IpeaGEO sintetiza e facilita o estudo e aplicação de técnicas econométricas espaciais.

10 Software do Centro de Ciências Espaciais da Universidade de Chicago. Disponível em: $<$ https://spatial.uchicago.edu/software $>$.
} 
São Paulo e Paraná) e dois países sul-americanos (Paraguai e Bolívia). A distribuição dos 79 municípios é apresentado na Figura 2. Para facilitar a análise da distribuição espacial dos municípios se utilizará as regiões de MS, a saber: Pantanal, Costa Leste, Vale do Aporé, Caminho dos Ipês, Bonito/Serra da Bodoquena, Caminhos da Fronteira, Vale das Águas, Grande Dourados, Cone Sul e Rota Norte.

Figura 2 - Mapa das regiões do Mato Grosso do Sul - MS

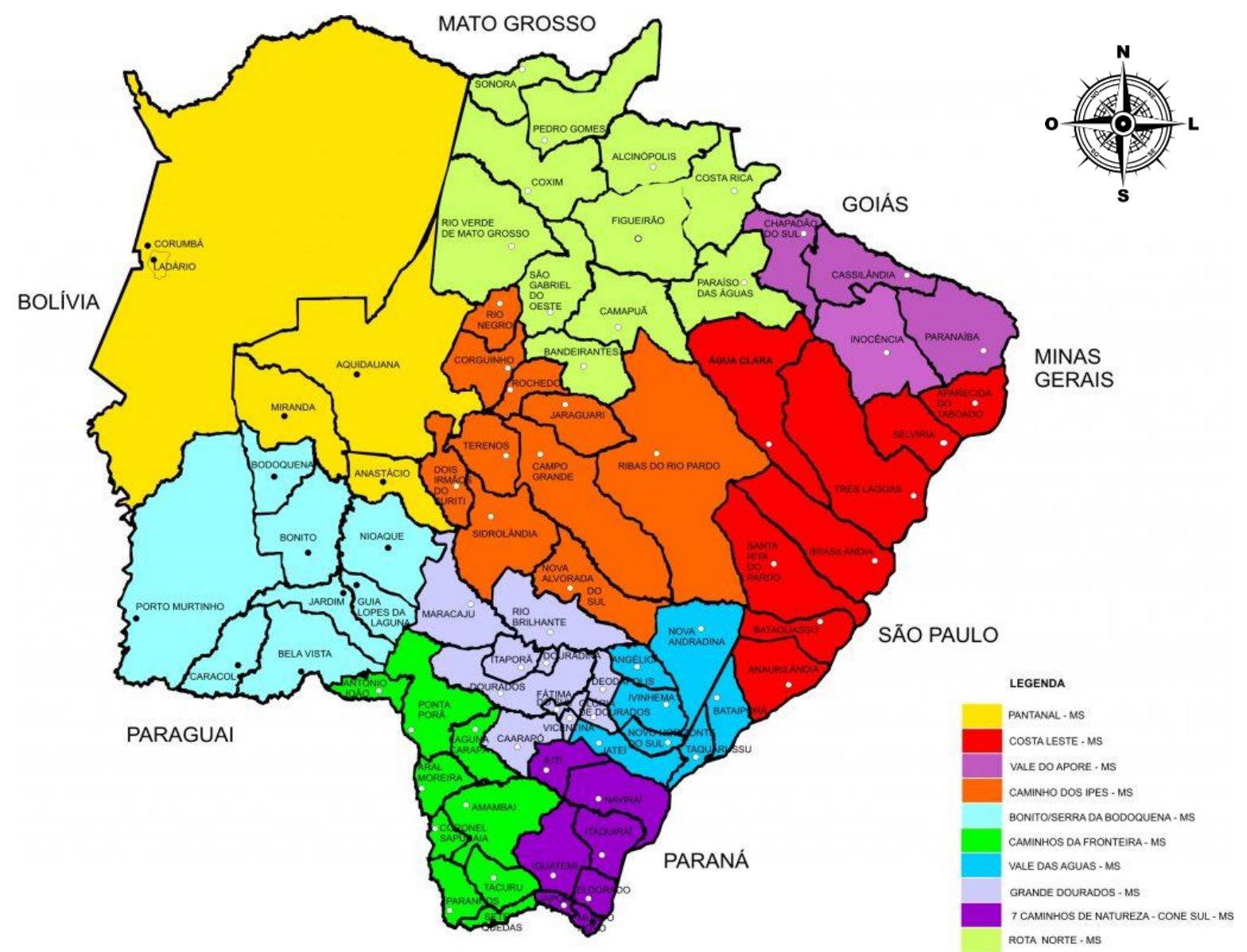

Fonte: Pantanal MS (2018)

Disponível em: <https://www.pantanalms.com.br/mapa-do-mato-grosso-do-sul-ms-brasil-turismo/>. Acesso em: 21 de agosto de 2019.

\section{Análise e discussão dos resultados}

Distribuição Espacial dos indicadores de Desenvolvimento Socioeconômico

Primeiramente, apresenta-se a evolução da distribuição espacial do IDHM e IFDM utilizando cinco quartis para relacionar os municípios conforme o nível do seu indicador, como pode ser observado a seguir (Figuras 3 a 6 ). 
Como pode-se observar na (Figura 3), para o IDHM de 1991, a região da Grande Dourados ${ }^{11}$ concentra o maior número de municípios dos dois quartis com maior IDHM, indicando que essa região apresenta maiores indicadores de desenvolvimento socioeconômico, o que pode ser um primeiro indício de que existe uma relação espacial de vizinhança entre municípios com alto IDHM. A mesma relação pode ser observada para os municípios da região Vale do Aporé ${ }^{12}$ de $M S$, entre os municípios de Chapadão do Sul, Cassilândia, Paranaíba e Aparecida do Taboado. É fácil perceber que cerca de 19\% dos municípios (quatorze) apresentaram IDHM superior a 0,47 estando estes no quartil de alto desenvolvimento humano, enquanto no outro extremo os municípios com menor desenvolvimento humano apresentaram IDHM inferior a cerca de 0,35 (19\% dos municípios).

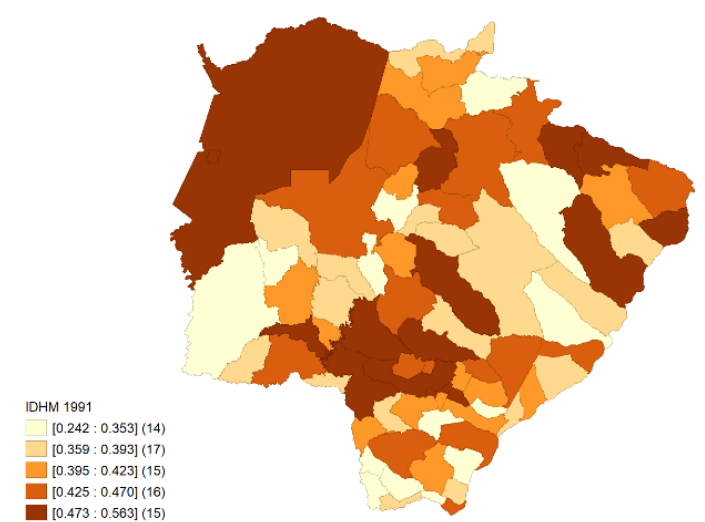

Figura 3. IDHM de MS em 1991 Fonte: Resultados da pesquisa.

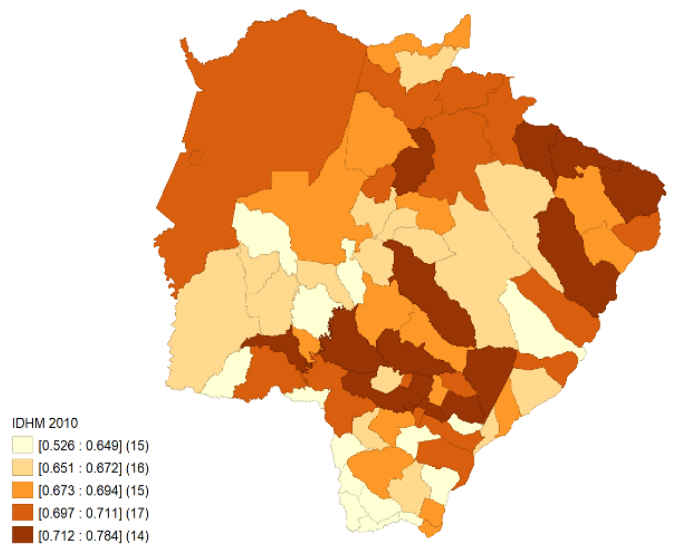

Figura 5. IDHM de MS em 2010 Fonte: Resultados da pesquisa.

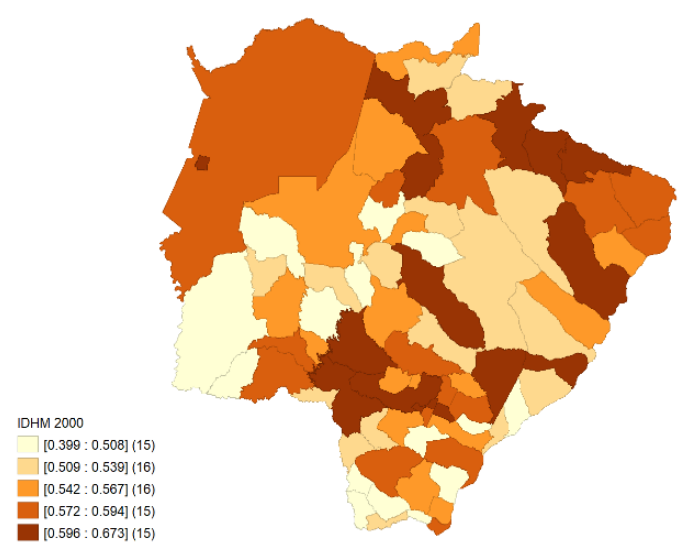

Figura 4. IDHM de MS em 2000 Fonte: Resultados da pesquisa.

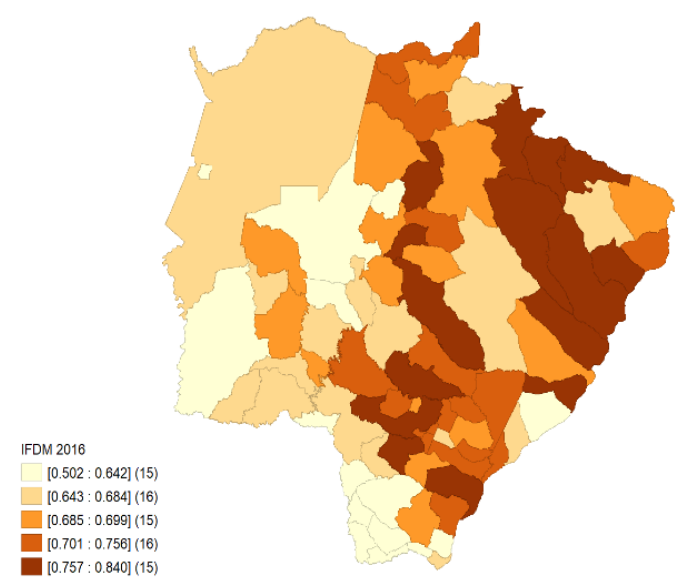

Figura 6. IFDM de MS em 2016 Fonte: Resultados da pesquisa.

\footnotetext{
${ }^{9}$ A região da Grande Dourados é formada pelos municípios de Dourados, Maracaju, Rio Brilhante, Itaporã, Douradina, Deodápolis, Fátima do Sul, Glória de Dourados e Vicentina.

12 O Vale do Aporé é formado pelos municípios de Chapadão do Sul, Cassilândia, Inocência, Paranaíba.
} 
$\mathrm{Na}$ Figura 4, para o IDHM de 2000, as regiões que apresentam melhores IDHM são Maracaju, Rio Brilhante, Dourados, Caarapó, Deodápolis e Glória de Dourados, municípios pertencentes a Região da Grande Dourados ${ }^{9}$. Cabe destacar também, Campo Grande Bataguassu, Nova Andradina, Coxim São Gabriel do Oeste, Costa Rica, Chapadão do Sul, Cassilândia, Paranaíba, Três Lagoas e Ladário que aparecem, também, com IDHM Alto.

Como pode-se observar no mapa da Figura 4, há indício de que existe uma relação espacial de vizinhança entre municípios com alto IDHM, dado que existem grupos de municípios de uma mesma região com alto IDHM. No outro extremo, as cidades de Bodoquena, Porto Murtinho, Caracol, Miranda, Nioaque, Miranda, Dois Irmãos do Buriti, Corguinho, Jutí, Itaquiraí, Nova Alvorada do Sul, Bataiporã, Coronel Sapucaia, Tacuru, Paranhos, Japorã são os quinze municípios que apresentaram menor IDHM, no entanto, em sua maioria, não estão localizadas na mesma região.

Para o IDHM de 2010 (Figura 5), os municípios que apresentam maior IDHM são Chapadão do Sul, Cassilândia, Paranaíba, Três Lagoas, São Gabriel do Oeste, Campo Grande, Maracaju, Rio Brilhante, Dourados, Caarapó, Jardim, Nova Andradina e Ivinhema. Alguns municípios apresentaram melhora significativa no aumento do seu IDHM, assim como seus vizinhos de municípios com IDHM alto. Os piores resultados apresentados, compõe os municípios de Miranda, Nioaque, Coronel Sapucaia, Tacuru, Paranhos, Japorã e Caracol que apresentaram o mesmo resultado do ano (2000), seguido dos municípios de Dois Irmãos do Buriti, Nova Alvorada do Sul, Jutí, Sete Quedas e Santa Rita do Rio Pardo.

Quanto aos melhores resultados apresentados de IFDM para o ano de 2016 (Figura 6), foram obtidos pelos municípios de Água Clara, Três Lagoas, Selviria, Brasilândia, Paraíso das Águas, Chapadão do Sul, Cassilândia, Rochedo, Campo Grande, Bataguassu, Rio Brilhante, Dourados, Naviraí e Caarapó, o que evidência, certa forma, a aglomeração de alguns municípios dado sua localização dentro de uma mesma região. Cabe destacar que os dois maiores municípios de MS, em termos populacionais, Dourados e Campo Grande, obtiveram índices positivos de crescimento desde (1991). Os piores resultados apresentados, são os municípios de Porto Murtinho, Aquidauana, Rio Negro, Anastácio, Ladário, Antônio João, Aral Moreira, Coronel Sapucaia, Tacuru, Amambai, Paranhos, Sete Quedas, Japorã e Anaurilândia, podendo-se perceber um número significativo de municípios da região Caminhos da Fronteira localizados no quartil de menor IFDM. 
Desta forma, quanto ao IFDM, cabe destacar os municípios de São Gabriel do Oeste $(0,8401)$ que ficou com a primeira posição no ranking, Três Lagoas $(0,8210)$ em segundo e Rio Brilhante $(0,8189)$ em terceiro, sendo que os três municípios são classificados como de alto estágio de desenvolvimento. Os piores resultados ficaram com Sete Quedas $(0,5597)$, Paranhos $(0,5584)$ e em último lugar Tacuru $(0,5022)$, todos classificados como desenvolvimento regular.

Analisando de forma mais minuciosa os dados para o IFDM de 2016, pode-se visualizar as variáveis Emprego e Renda, Saúde e Educação (ver Figuras 12, 13 e 14 no Apêndice A). Os municípios que apresentaram os melhores resultados de IFDM para Emprego e Renda no MS foram, respectivamente, Selvíria $(0,8002)$, Água Clara $(0,7925)$ e Dourados $(0,7668)$, enquanto os piores índices ficaram com os municípios de Sete Quedas $(0,3236)$, Ladário $(0,3218)$ e Eldorado $(0,3127)$. Para - IFDM Saúde os melhores municípios foram Vicentina $(0,9593)$, seguido de Rio Brilhante $(0,9395)$ e Alcinópolis $(0,9257)$, e os piores resultados foram para os municípios de Paranhos (0,5727), Antônio João $(0,5560)$ e, por último, Tacuru $(0,4578)$. Por fim, para o IFDM Educação os municípios com melhores índices foram Costa Rica $(0,8968)$, Taquarussu $(0,8968)$ e Cassilândia $(0,8775)$, enquanto os piores resultados ficaram com Porto Murtinho $(0,0656)$, Paranhos $(0,6296)$ e, o pior colocado, Tacuru $(0,5968)$.

Pode-se perceber com bases nas Figuras 3 à 6, que houve mudança na região do Pantanal $^{13}$ quanto a classificação de alguns dos seus municípios, que inicialmente figuravam no quartil de mais elevado IDHM, passando para os dois últimos quartis de menor IDHM em 2016, caso dos municípios de Corumbá, Ladário e Aquidauana. Cabe destacar também, de forma negativa, a região Caminhos da Fronteira $^{14}$ a qual apresenta o maior número de municípios no quartil de menor IFDM em 2016. De maneira oposta as Regiões da Grande Dourados, Vale do Aporé e Costa Leste ${ }^{15}$ são as que apresentam maior número de municípios no quartil de maior IDHM, sendo que as mesmas apresentaram um padrão de alto IDHM ao longo dos períodos analisados.

\footnotetext{
${ }^{13}$ Região do Pantanal é formada pelos municípios de Corumbá, Ladário, Aquidauana, Miranda e Anastácio.

14 Região Caminhos da Fronteira é formado pelos municípios de Antônio João, Aral Moreira, Amambai, Laguna Carapã, Coronel Sapucaia, Tacuru, Paranhos, Ponta Porã e Sete Quedas.

15 Costa Leste é composta pelos municípios de Água Clara, Três Lagoas, Selvíria, Aparecida do Taboado, Brasilândia, Santa Rita do Rio Pardo, Bataguassu e Anaurilândia.
} 
Resultados da AEDE

Para realizar a análise de cluster, como descrito anteriormente, é necessário evidenciar a existência de um padrão espacial nos dados, para isso estima-se o Índice de Moran Global que mostra a autocorrelação espacial, usando diferentes matrizes de pesos espaciais visando identificar a que possui melhor ajuste com o padrão espacial, caso o mesmo exista.

Como pode ser observado na Tabela 1, a maior parte das matrizes apresentou autocorrelação espacial (I de Moran) positiva e significativa, indicando a existência de um padrão espacial nos dados, cujas dispersões para as matrizes com melhor robustez são apresentadas na (Figura 7).

Para a análise da associação espacial (LISA) ou análise de formação de cluster entre os municípios, se utiliza a matriz que demonstrou melhor robustez, significância estatística, para cada variável em estudo, sendo a matriz de ordem $K=$ 5 (cinco vizinhos mais próximos ou vizinhança de quinta ordem) a que apresentou melhor ajuste para a maior parte das variáveis.

Tabela 1. Índice de Moran Global (Autocorrelação)

\begin{tabular}{c|ccccccc}
\hline $\begin{array}{c}\text { Matriz/ } \\
\text { Variável }\end{array}$ & IDHM 1991 & IDHM 2000 & IDHM 2010 & IFDM 2016 & Emp. e Renda & Educação & Saúde \\
\hline K1 & $0,204^{*}$ & 0,257 & 0,324 & 0,542 & 0,287 & 0,357 & 0,557 \\
& $(0,064)$ & $(0,020)$ & $(0,008)$ & $(0,001)$ & $(0,014)$ & $(0,005)$ & $(0,001)$ \\
K3 & 0,105 & 0,152 & 0,287 & 0,416 & 0,118 & 0,308 & 0,469 \\
& $(0,078)$ & $(0,026)$ & $(0,001)$ & $(0,001)$ & $(0,075)$ & $(0,002)$ & $(0,001)$ \\
K5 & 0,061 & $0,158^{*}$ & $0,31^{*}$ & $0,436^{*}$ & 0,162 & $0,294^{*}$ & $0,441^{*}$ \\
& $(0,127)$ & $(0,010)$ & $(0,001)$ & $(0,001)$ & $(0,007)$ & $(0,001)$ & $(0,001)$ \\
Rook & 0,035 & 0,103 & 0,249 & 0,254 & 0,045 & 0,201 & 0,216 \\
& $(0,159)$ & $(0,016)$ & $(0,001)$ & $(0,001)$ & $(0,122)$ & $(0,001)$ & $(0,001)$ \\
Queen & 0,071 & 0,154 & 0,307 & 0,0461 & $0,191^{*}$ & 0,332 & 0,444 \\
& $(0,112)$ & $(0,016)$ & $(0,001)$ & $(0,001)$ & $(0,005)$ & $(0,001)$ & $(0,001)$ \\
\hline
\end{tabular}

Nota: Valores da probabilidade entre parênteses ( $p$-value). ${ }^{*}$ Coeficientes com maior significância estatística.

Fonte: Resultados da pesquisa. 
Figura 7 - Dispersão do Índice de Moran Global (/)
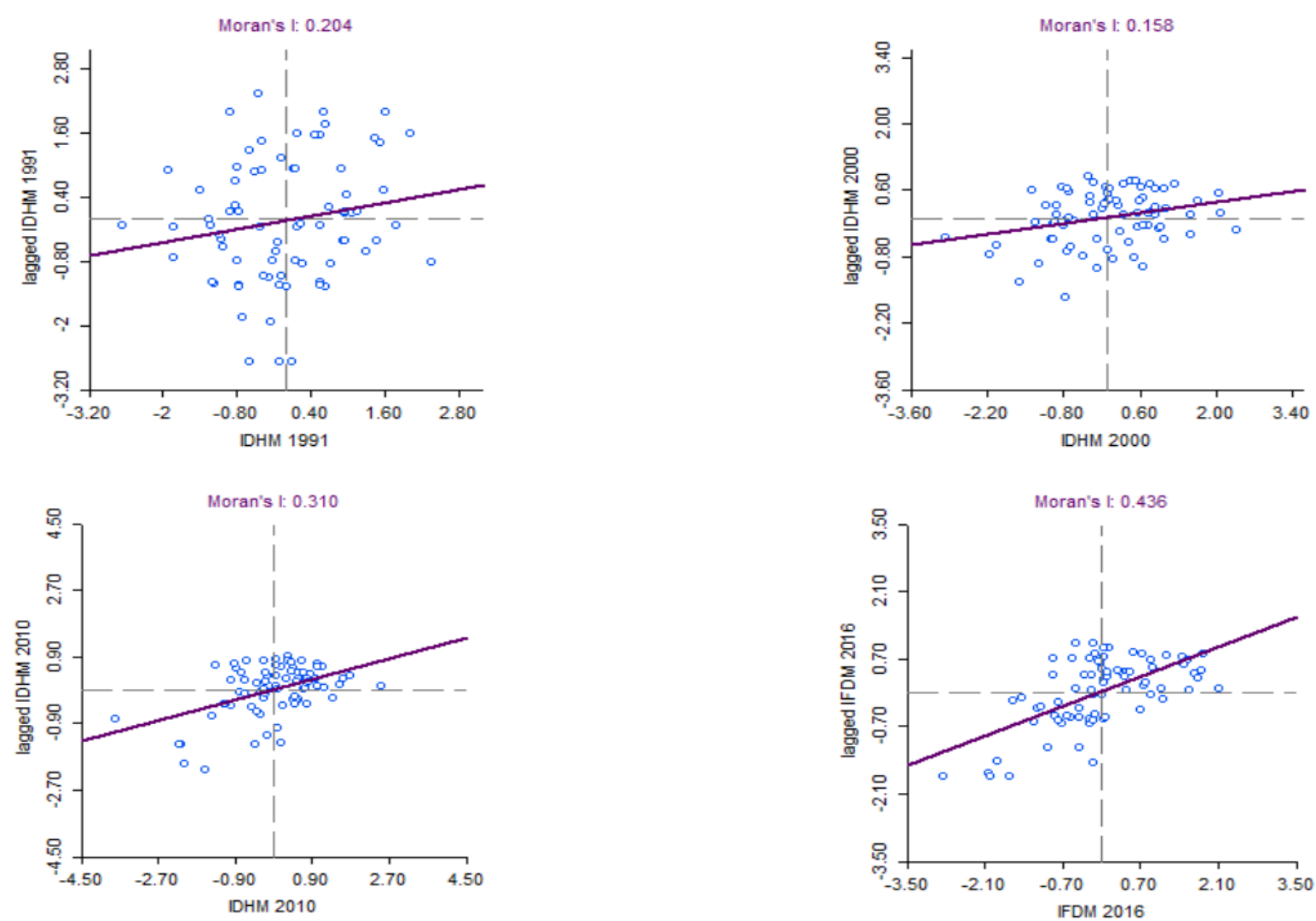

Fonte: Resultados da pesquisa.

As dispersões, apresentadas na Figura 7, evidenciam a dependência espacial nos dados, de modo que existe uma similaridade ou padrão na estrutura dos mesmos, portanto essa relação precisa ser considerada. A seguir, apresenta-se o resultado do LISA para a evolução do IDHM, de 1991 a 2010, e o IFDM de 2016.

Figura 8 - Indicador LISA para o IDHM $1991(k=1)$

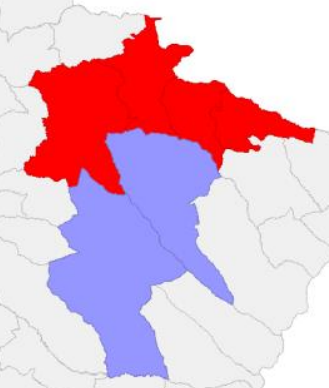


Nota: Vermelho (Alto-Alto); Azul (Baixo-Baixo); Azul claro (Baixo-Alto); Rosa (Alto-Baixo); Cinza (Não significativo).

Fonte: Resultados da pesquisa.

Como pode-se observar na Figura 8, o LISA apresentou a formação de quatro clusters de IDHM para o ano de 1991. O cluster Alto-Alto (AA) é formado pelos municípios Costa Rica, Paraíso das Águas, Chapadão do Sul e Cassilândia. Essa relação indica que a localização geográfica desses municípios e seus vizinhos é importante para o nível de desenvolvimento humano local, sendo que tais municípios apresentam alto IDHM e são cercados por municípios com alto IDHM. O cluster Baixo-Baixo (BB) é formado pelos municípios Jateí, Taquárussu e Iguatemi. Ou seja, tais municípios apresentam baixo IDHM e são cercados por municípios com baixo IDHM. O cluster Baixo-Alto (BA) é constituído pelos municípios Água Clara e Ribas do Rio Pardo. Esse resultado mostra que tais municípios apresentam baixo IDHM e são cercados por municípios com alto IDHM. O cluster Alto-Baixo (AB) é formado pelo município de Ivinhema. Portando, o município de Ivinhema apresenta alto IDHM e é rodeado por municípios com baixo IDHM.

Figura 9 - Indicador LISA para o IDHM $2000(k=5)$
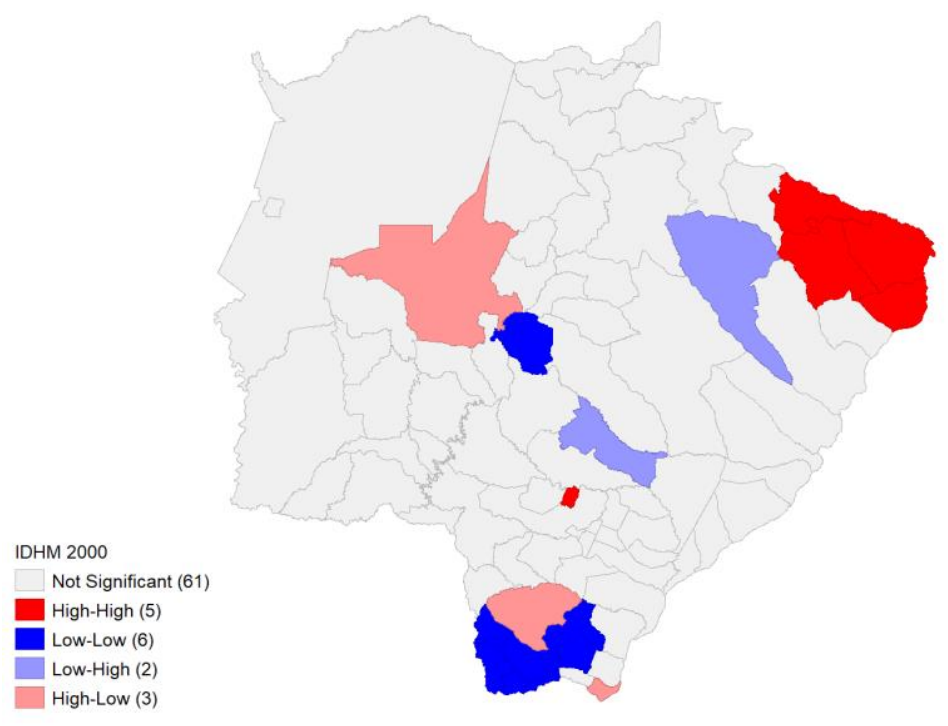

Nota: Vermelho (Alto-Alto); Azul (Baixo-Baixo); Azul claro (Baixo-Alto); Rosa (Alto-Baixo); Cinza (Não significativo).

Fonte: Resultados da pesquisa.

Na Figura 9, considerando o IDHM de 2000, o LISA apresentou aglomerações significativas para dezesseis municípios. O cluster AA é formado pelos municípios Cassilândia, Paranaíba, Inocência, Aparecida do Taboado e Douradina. Esses municípios pertencem a região do Vale do Aporé, com exceção apenas de 
Aparecida do Taboado e Douradina, indicando que o IDHM alto desses municípios possui relação com os seus municípios vizinhos também possuírem alto IDHM. 0 cluster BB é formado pelos municípios Coronel Sapucaia, Paranhos, Tacuru, Iguatemi e Sete Quedas, enquanto o município de Terenos compõe outra aglomeração BB por estar perto de um município pertencente ao cluster AB. Esses municípios (BB) apresentam baixo IDHM e são cercados por municípios com baixo IDHM. O cluster BA é constituído pelos municípios Nova Alvorada do Sul e Água Clara. Esse resultado mostra que tais municípios apresentam baixo IDHM e são cercados por municípios com alto IDHM. O cluster AB é formado pelo município de Aquidauana, Amambai e Mundo Novo.

Pode-se observar (Figura 10), que o LISA apresentou a formação de clusters de IDHM para o ano de 2010. O cluster AA é formado por seis municípios (Costa Rica, Inocência, Aparecida do Taboado, Nova Alvorada do Sul, Deodápolis e Glória de Dourados), indicando relação positiva e de alto IDHM entre esses municípios e seus vizinhos que também apresentaram resultado significativo (fazem parte do mesmo agrupamento). Essa relação indica que a localização geográfica desses municípios e seus vizinhos é importante para o nível de desenvolvimento humano local, sendo que tais municípios apresentam alto IDHM e são cercados por municípios com alto IDHM.

Figura 10 - Indicador LISA para o IDHM $2010(k=5)$
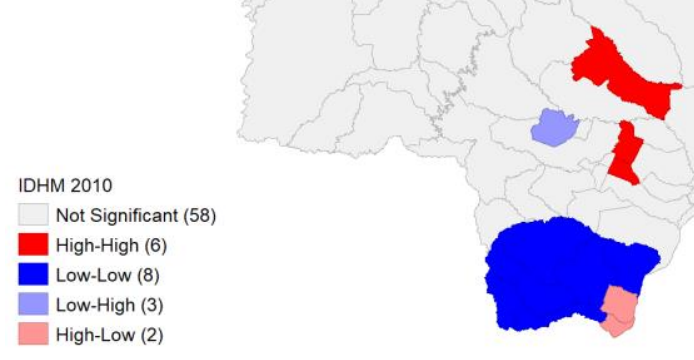

Nota: Vermelho (Alto-Alto); Azul (Baixo-Baixo); Azul claro (Baixo-Alto); Rosa (Alto-Baixo); Cinza (Não significativo).

Fonte: Resultados da pesquisa. 
O cluster BB é formado pelos municípios de Amambai, Coronel Sapucaia, Paranhos, Tacuru, Corguinho, Iguatemi, Itaquirai e Sete Quedas. Esses municípios apresentam baixo IDHM e são rodeados por municípios com baixo IDHM. O cluster BA é constituído pelos municípios Itaporã, Santa Rita do Pardo e Água Clara. Esse resultado mostra que tais municípios apresentam baixo IDHM e são cercados por municípios com alto IDHM. O cluster $A B$ é formado pelos municípios de Eldorado e Mundo Novo (Figura 10).

Analisando o IFDM para o ano de 2016 (Figura 11), O LISA apresentou a formação de clusters significativo de apenas três tipos (AA, BB e BA). O cluster AA é formado por nove municípios (Costa Rica, Cassilândia, Paranaíba, Aparecida do Taboado, Três Lagoas, Água Clara, Nova Alvorada Do Sul, Santa Rita do Rio Pardo e Itaporã), indicando que o número de municípios com relação significativa para o cluster AA teve aumento, evidenciando que o alto IFDM nesses municípios depende positivamente do alto IFDM de seus vizinhos. O cluster BB é formado por oito municípios (Amambai, Paranhos, Tacuru, Coronel Sapucaia e Sete Quedas, da região Caminhos da Fronteira, e Iguatemi, Mundo Novo e Japorã, da região 7 Caminhos de Natureza), indicando que municípios com baixo IFDM são rodeados por municípios com também baixo IFDM, mostrando a existência de uma relação significativa entre esses municípios, de que ter vizinhos com baixo IFDM influencia em possuir baixo IFDM. O cluster BA é formado pelos municípios de Paraíso das Águas, Inocência, Sidrolândia e Douradina, mostrando que os mesmos possuem baixo IFDM e são cercados por municípios com alto IFDM.

Figura 11 - Indicador LISA para o IFDM $2016(k=5)$
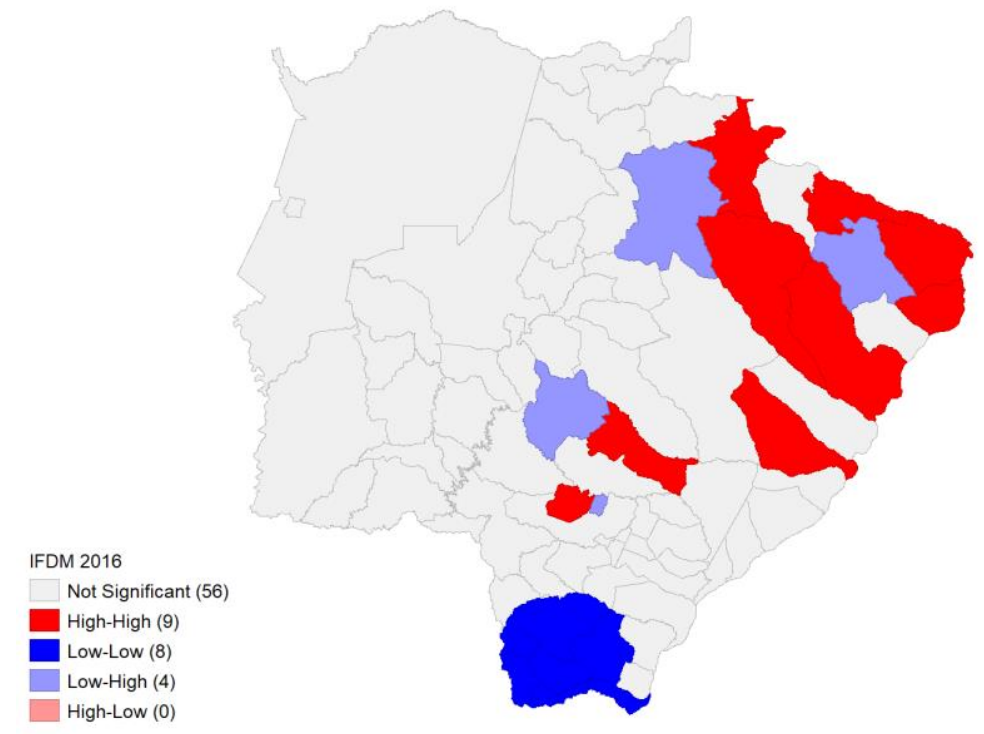
Nota: Vermelho (Alto-Alto); Azul (Baixo-Baixo); Azul claro (Baixo-Alto); Rosa (Alto-Baixo); Cinza (Não significativo).

Fonte: Resultados da pesquisa.

A análise de cluster (LISA), como pode-se observar (Figuras 8 à 11), demonstra a existência de diferenças regionais quanto ao desenvolvimento socioeconômico para os municípios de MS. Essa diferença é evidenciada principalmente pela formação de clusters do tipo AA e BB para diferentes regiões do estado, que mostram que para tais municípios a sua localização espacial é importante para explicar o seu nível de IDHM, dado que municípios com alto IDHM são cercados por municípios com alto IDHM (cluster AA nos mapas), e o contrário também é verdadeiro (cluster BB nos mapas). As regiões em destaque com maior número de clusters do tipo AA foram Costa Leste ${ }^{4}$ e Vale do Aporé, enquanto a região Caminhos da Fronteira apresentou cluster do tipo BB para IFDM, sendo que de forma individualizada para emprego e renda, educação e saúde, também evidenciou-se a formação de cluster BB para essa região (Figuras 15 à 17 do Apêndice A). A região Caminhos da Fronteira, apresentou a maior parte de seus municípios presentes no cluster BB a partir do ano 2000, com aumento de municípios a cada ano analisado, o que indica uma necessidade de políticas públicas específicas para essa região buscando reduzir as disparidades regionais quanto ao desenvolvimento socioeconômico na mesma, comparado a regiões como o Vale do Aporé e Costa Leste.

Quando analisado de forma individualizada o IFDM de 2016, quanto a saúde (ver Figura 16 no Apêndice A), verifica-se dois importantes clusters do tipo AA para Região da Grande Dourados e Rota Norte ${ }^{16}$, o que evidência a existência de melhor qualidade da saúde nos municípios que formam os mesmos. Isso, de certa forma, evidencia a importância do município de Dourados como importante centro de saúde para a Região da Grande Dourados.

\section{Considerações finais}

Este trabalho teve como objetivo analisar a possível existência de diferenças regionais quanto ao desenvolvimento socioeconômico nos municípios de Mato Grosso do Sul. Para tanto, utilizou-se o ferramental da Análise Exploratória de

\footnotetext{
${ }^{16}$ Rota Norte é formada por Sonora, Pedro Gomes, Coxim, Rio Verde, Alcinópolis, Figueirão, Costa Rica, Paraiso das Águas, São Gabriel do Oeste, Bandeirantes e Camapuã.
} 
Dados Espaciais e as variáveis do IDHM para os anos de 1991, 2000 e 2010 e o IFDM para o ano de 2016, buscando verificar a existência de clusters espaciais.

Quanto a distribuição espacial dos indicadores analisados, verificou-se que houve mudança negativa na região do Pantanal quanto a classificação de alguns dos seus municípios, que inicialmente figuravam no quartil de mais elevado IDHM, passando para quartis de menor IFDM em 2016. A região Caminhos da Fronteira foi a que apresentou o maior número de municípios no quartil de menor IFDM em 2016. Cabe destacar também, positivamente, as Regiões da Grande Dourados, Vale do Aporé e Costa Leste, como as que apresentam maior número de municípios no quartil de maior IFDM, sendo que as mesmas apresentaram um padrão de alto IDHM ao longo dos períodos analisados.

A análise de formação de clusters, demonstrou a existência de diferenças regionais quanto ao desenvolvimento socioeconômico para os municípios de MS, sendo evidenciada principalmente pela formação de clusters de municípios com alto desenvolvimento socioeconômico cercadas por municípios com alto desenvolvimento socioeconômico, enquanto o oposto ocorreu para diferentes regiões do estado, que mostram que para tais municípios a sua localização espacial é importante para explicar o seu nível de desenvolvimento humano. As regiões em destaque com maior número de clusters de alto desenvolvimento socioeconômico foram Costa Leste e Vale do Aporé, enquanto a região Caminhos da Fronteira apresentou cluster de baixo desenvolvimento socioeconômico, principalmente a partir do ano de 2000 com número crescente de municípios nos anos subsequentes, sendo que, para essa região, de forma individualizada para emprego e renda, educação e saúde, também evidenciou-se a formação de cluster do tipo BaixoBaixo. Esse resultado indica uma necessidade de soluções específicas para essa região, buscando reduzir as disparidades regionais, comparado a regiões como o Vale do Aporé e Costa Leste.

Por fim, verificada a existência de diferenças regionais quanto ao desenvolvimento socioeconômico e a formação de clusters de municípios com baixo DS e de municípios com alto DS, fica clara a necessidade de políticas públicas voltadas para sanar essas disparidades. Fica o desafio, para trabalhos futuros, identificar quais são os principais fatores que levaram a um desenvolvimento regional desequilibrado, na tentativa de apontar possíveis caminhos mais específicos para as políticas públicas. 


\section{Referências}

ALMEIDA, E. Econometria Espacial. Campinas-SP. Alínea, 2012.

ALMEIDA, E. S. Curso de Econometria Espacial Aplicada: Matrizes de Pesos Espaciais. 2004. Curso de Econometria Espacial, Departamento de Economia, Administração e Sociologia da Esalq-USP, Piracicaba, 2004.

ANSELIN, L. Spatial econometrics, 1999. Disponível em: <http://www.csiss.org/learning_resources/content/papers/baltchap.pdf>. Acesso em: 16 de junho de 2019.

ARAUJO, E.; URIBE-OPAZO, M. A.; JOHANN, J. A. Modelo de regressão espacial para estimativa da produtividade da soja associada a variáveis agrometeorológicas na região oeste do estado do Paraná. Eng. Agrícola. Jaboticabal, v.34, n.2, mar./abr. de 2014.

BEATO C. C. F.; REIS I. A. Desigualdade, desenvolvimento socioeconômico e crime. Departamento de Sociologia e Antropologia da UFMG, 2015.

CONSTANTINO, M.; PEGORARE B. A.; COSTA B. R. Desempenho regional do IDH e do PIB per capita dos municípios de Mato Grosso do Sul, Brasil, entre 2000 e 2010. Interações, Campo Grande - MS, v. 17, n. 2, p. 234-246, abr./jun. 2016.

FIRJAN. Índice FIRJAN de Desenvolvimento Municipal (IFDM). Ano base 2016, ano 2018. Recorte Municipal de Abrangência Nacional - Emprego e Renda Saúde e Educação. Disponível em: <www.firjan.com.br/ifdm/>. Acesso em: 30 de outubro de 2019.

GADELHA, C. A. G.; MALDONADO, J. M. S. V.; COSTA, L. S. O complexo produtivo da saúde e sua relação com o desenvolvimento: um olhar sobre a dinâmica da inovação em saúde. Revista do Serviço Público Brasília, abr/jun 2013.

HOECKEL, P. H. O.; CASAGRANDE, D. L.; SANTOS, C. A. P. Análise Espacial da Pobreza no Rio Grande do Sul. In: FOCHEZATTO, A.; PELEGRINI, T.; HOECKEL, P. H.;

TOMKOWSKI, F. G. (Org.). Desenvolvimento Socioeconômico Regional: cidades, crescimento e especialização produtiva. 1ed.Porto Alegre: EDIPUCRS, 2019, v. 1, p. 129-160.

IBGE. Instituto Brasileiro de Geografia e Estatística. Pesquisa Nacional por Amostra de Domicílios: desenvolvimento socioeconômico. Mato Grosso do Sul, 2010. Disponível em: $<$ http://www.estatisica.ibge.gov.br>. Acesso em: 20 de maio de 2019.

IDHM. Índice de Desenvolvimento Humano Municipal Brasileiro. Brasília: PNUD, Ipea, FJP, 2013. 96 p. - (Atlas do Desenvolvimento). Disponível em: <http/www.atlasbrasil.org.br>. Acesso em: 26 de abril de 2019.

IPEA. Instituto de Pesquisa Econômica Aplicada: Instituto de Pesquisa Econômica Aplicada, 2018. Disponível em: <http://www.ipea.gov.br>. Acesso em: 10 de março de 2019.

LIMA, J. F; PIFFER, M.; OSTAPECHEN, L. A. P. O crescimento econômico regional de Mato Grosso do Sul. Interações, Campo Grande, MS, v. 17, n. 4, p. 757-766, out./dez. 2016.

MOLDAU, H. J. Os fundamentos microeconômicos dos indicadores de desenvolvimento socioeconômico. Universidade de São Paulo, FEA - USP, 1998.

MORAES, F. Universidade, Inovação e Impacto Socioeconômico. São Paulo em Perspectiva, v.14, n.3, 2000. 
PAES DE BARROS R.; MENDONÇA R. Investimentos em Educação e Desenvolvimento Econômico, Rio de Janeiro, novembro de 1997.

PANTANAL MS, 2018. Mapa do Mato Grosso do Sul - MS - Brasil. Emerson Brito (Org.). Disponível em: <https://www.pantanalms.com.br/mapa-do-mato-grosso-do-sul-msbrasil-turismo/>. Acesso em: 21 de agosto de 2019.

PERES, M. F. T.; CARDIA, N.; MESQUITA NETO, P. D.; SANTOS, P. C. D.; ADORNO, S. Homicídios, desenvolvimento socioeconômico e violência policial no município de São Paulo, Brasil. Revista Panamericana de Salud Publica, 23, 268-276, 2008.

PNUD. Programa Nacional das Nações Unidas para o Desenvolvimento. Atlas Brasil, 2013. [s.d.]. Disponível em: <www.pnud.org.br>. Acessado em: 23 de maio de 2019.

REIS, P. R. C.; SILVEIRA, S. F. R.; BRAGA, M. J. Previdência social e desenvolvimento socioeconômico: impactos nos municípios de pequeno porte de Minas Gerais. Revista de Administração Pública, v. 47, n. 3, p. 623-646, 2013.

REZENDE, A. J.; SLOMSKI, V.; CORRAR, L. J. A gestão pública municipal e a eficiência dos gastos públicos: uma investigação empírica entre as políticas públicas e o Índice de Desenvolvimento Humano (IDH) dos municípios do estado de São Paulo. Revista Universo Contábil, v. 1, n. 1, p. 24-40, 2005.

ROCHA, S. Desigualdade Regional e Pobreza no Brasil: A Evolução - 1981/95, Rio de Janeiro, junho de 1998.

RODRIGUES, C. G.; SIMÕES, R. F. Aglomerados industriais e desenvolvimento socioeconômico: uma análise multivariada para Minas Gerais. Ensaios FEE, Porto Alegre, v. 25, n. 1, p. 203-232, abr. 2004.

SEN, A. Desenvolvimento como Liberdade. São Paulo: Companhia das Letras, 2000.

SHIKIDA F. A. P. Crimes violentos e desenvolvimento socioeconômico: um estudo para o estado do Paraná. Direitos fundamentais \& justiça, n.5, out./dez., 2008.

SIEDENBERG, D. R. Indicadores de desenvolvimento socioeconômico: uma síntese. Desenvolvimento em Questão, v. 1, n. 1, p. 45-71, 2003.

SICSÚ, J.; CASTELAR, A. (Org.) Sociedade e Economia: estratégias de crescimento e desenvolvimento. Brasília: (2009) 256 f. Instituto de Pesquisa Econômica Aplicada. Disponível em: <http://www.ipea.gov.br>. Acesso em: 10 de março de 2019.

VIDIGAL, V. G.; AMARAL, I. C.; SILVEIRA, G. F. Desenvolvimento socioeconômico nas microrregiões do Paraná: Análise Multivariadas. Revista de Economia, v. 38, n. 2 (ano 36), p. 51-72, maio/ago. 2012. Editora UFPR. 


\section{APÊNDICE A}






\section{CONTRIBUIÇÃO DE AUTORIA}

\section{NOTAS DE AUTOR}

Paulo Henrique Hoeckel - Concepção e elaboração do manuscrito. Coleta e análise de dados. Participação ativa da discussão dos resultados. Revisão e aprovação da versão final do trabalho.

Maria Cristina Vezzu Costa - Concepção e elaboração do manuscrito. Análise de dados. Participação ativa da discussão dos resultados. Aprovação da versão final do trabalho.

\section{FINANCIAMENTO}

Não se aplica.

\section{CONSENTIMENTO DE USO DE IMAGEM}

Não se aplica.

\section{APROVAÇÃO DE COMITÊ DE ÉTICA EM PESQUISA}

Não se aplica.

\section{CONFLITO DE INTERESSES}

Não se aplica.

\section{LICENÇA DE USO}

Este artigo está licenciado sob a Licença Creative Commons CC-BY. Com essa licença você pode compartilhar, adaptar, criar para qualquer fim, desde que atribua a autoria da obra.

\section{HISTÓRICO}

Recebido em: 20-07-2020

Aprovado em: 02-03-2021 